\title{
ACCOUNTABILITY: UMA OBSERVAÇÃO SOBRE O NÍVEL DE TRANSPARÊNCIA DE MUNICÍPIOS
}

\author{
Accountability: a Note on the Level of Transparency of Municipalities \\ Accountability: una Observación sobre el Nivel de Transparencia de Municipios
}

Filipy Furtado Sell Doutorando em Contabilidade na Universidade Federal de Santa Catarina (UFSC) http://lattes.cnpq.br/7867452877557402 professor@sellcontabilidade.com.br

Graciele Lima Sampaio Mestre em Ciências Contábeis pela Universidade Regional de Blumenau - FURB Professora da Universidade Católica de Pelotas - UCPEL e Professora Substituta da Universidade Federal do Rio Grande - FURG http://lattes.cnpq.br/0749775406490015 https://orcid.org/0000-0001-6943-6484 graciele_sampaio@yahoo.com.br

Vinícius Costa da Silva Zonatto Pós-doutor em Ciências Contábeis (UNISINOS) (Controle de Gestão) Doutor em Ciências Contábeis e Administração (FURB) (Controladoria) Professor Adjunto da Universidade Federal de Santa Maria http://lattes.cnpq.br/1916486402947867 https://orcid.org/0000-0003-0823-6774 viniciuszonatto@gmail.com

Carlos Eduardo Facin Lavarda

Doutorado em Contabilidade pela Universitat de Valencia, Espanha Bolsista CNPq Professor do Departamento de Ciências Contábeis http://lattes.cnpq.br/0990433322587449 http://orcid.org/0000-0003-1498-7881 eduardo.lavarda@ufsc.br

Resumo: O presente estudo tem por objetivo verificar o nível de accountability nos municípios da Região Sul do Brasil, segregando entre os 10 municípios mais e menos populosos de cada Estado, contemplando 60 municípios. Para identificar o nível de transparência utilizou-se a metodologia adaptada de Keunecke, Teles e Flach (2011) e Speck (2014), após verificou-se a correlação de Kendall para identificar se há relação entre o nível de accountability e os indicadores socioeconômicos e econômicofinanceiro dos municípios. Os achados demonstram que o maior nível de accountability foi em Joinville (SC) e o menor em Jardinópolis (SC); e dentre os Estados o maior nível de accountability foi do Paraná, seguido por Rio Grande do Sul e Santa Catarina, respectivamente. Quanto a relação entre os indicadores pode-se inferir que quanto maior o nível de Accountability nos municípios maior tende ser o níveis de Desempenho Socioeconômico e do Desempenho EconômicoFinanceiro.

Palavras-chave: Accountability; Setor Público; Municípios; New Public Management.

\begin{abstract}
The present study aims to verify the level of accountability in the municipalities of the Southern Region of Brazil, segregating among the 10 more and less populous cities of each State, contemplating 60 municipalities. In order to identify the level of transparency, the methodology adapted from Keunecke, Teles and Flach (2011) and Speck (2014) was used. After was verified the Kendall's correlation to identify if there is a relation between the level of accountability and socioeconomic indicators and economic-financial situation of the municipalities. The results show that the highest level of accountability was in Joinville (SC) and the lowest in Jardinópolis (SC); and among the states the highest level of accountability was from Paraná followed by Rio Grande do Sul and Santa Catarina, respectively. Regarding the relationship between indicators, it can be inferred that the higher the level of Accountability in the municipalities, the higher the level of Socioeconomic Performance and of EconomicFinancial Performance tend to be.
\end{abstract}

Keywords: Accountability; Public sector; Counties; New Public Management
Resumen: El presente estudio tiene por objetivo verificar el nivel de accountability en los municipios de la Región Sur de Brasil, segregando entre los 10 municipios más y menos poblados de cada Estado, contemplando 60 municipios. Para identificar el nivel de transparencia se utilizó la metodología adaptada de Keunecke, Teles y Flach (2011) y Speck (2014). Después de que se verificó la correlación de Kendall para identificar si existe relación entre el nivel de accountability y los indicadores socioeconómicos, económico y financiero de los municipios. Los hallazgos demuestran que el mayor nivel de accountability fue en Joinville (SC) y el menor en Jardinópolis (SC); y entre los Estados el mayor nivel de accountability fue de Paraná, seguido por Rio Grande do Sul y Santa Catarina, respectivamente. En cuanto a la relación entre los indicadores se puede inferir que cuanto mayor el nivel de Accountability en los municipios mayor tiende a ser el nivel de Desempeño Socioeconómico y el nivel del Desempeño Económico-Financiero.

Palabras clave: Accountability; Sector Público; Municipios; New Public Management.

\section{INTRODUÇÃO}

A New Public Management (NPM) orienta mudanças na gestão pública visando aumentar a eficiência e eficácia e dispender maiores esforços no processo de tomada de decisão com base em inovações de sistemas de gestão contábil e accountability (Parker \& Gould, 1999; Pérez-López, Prior \& Zafra-Gómez, 2015). Os autores ainda destacam que as mudanças são impulsionadas pela globalização, pelas propostas de privatizações, pelo governo cingindo uma filosofia de facilitador das prestações de serviços públicos, ao invés de prestador direto do serviço e pela absorção de conceitos da contabilidade privada para o gerenciamento da coisa pública.

Campbell (1993), Gore (1995), Peters e Savoie (1996), destacam que as mudanças propostas para a gestão pública visam

Correspondência/Correspondence: Filipy Furtado Sell. Universidade Federal de Santa Catarina (UFSC, Centro Socioeconômico, Rua Eng. Agronômico Andrei Cristian Ferreira, $\mathrm{s} / \mathrm{n}$ - Trindade, Florianópolis - SC, Brasil. CEP 88040-900 professor@sellcontabilidade.com.br 
aumentar a eficiência, eficácia, medição do desempenho, capacitação dos servidores, redução burocrática e elaboração de prestações de contas mais transparentes. Neste sentido, Romzek (2000) salienta que nesse processo de mudanças um dos principais desafios é a divulgação de mais transparência na prestação de contas dos gestores públicos. Van der Hoek (2005) destaca que a NPM facilitará a comparação entre os dados dos países visando melhorar a gestão e o desempenho, fortalecendo o controle das contas públicas e facilitando a responsabilização dos gestores pela má gestão dos recursos públicos.

Nesse contexto tem-se uma crescente utilização dos meios eletrônicos e da rede mundial de computadores para a promoção da accountability, tanto nas empresas privadas como nos órgãos e empresas do setor público, com o intuito de diminuir os dispêndios aplicados na disponibilização de informações para o controle social (Akutsu \& Pinho, 2002).

Observando os estudos de Cameron (2004), Schwella (2005) e Schillemans (2015), destaca-se como desafio e tendência de gestão para as lideranças públicas a busca da "boa governança" pública, compreendendo ações para fomentar a democratização da política, a transparência das ações governamentais e na accountability.

No Brasil, o Governo Federal, no ano de 2009, observando o cenário mundial referente à NPM, sancionou a Lei Complementar № 131, ampliando os ditames da Lei de Responsabilidade Fiscal (LRF). A ampliação define a disponibilização em tempo real dos dados concernentes à execução do orçamento e do financeiro, em "meios eletrônicos de acesso público", com objetivo de assegurar a transparência dos gastos públicos e a fiscalização social.

Nesse sentido, Keunecke et al. (2011) afirmam que a LC no 131/2009, intensifica as obrigações e responsabilidades, fazendo com que a administração pública preste contas dos recursos geridos e os órgãos responsáveis façam o devido acompanhamento (fiscalização). Bairral, Silva e Alves (2015) complementam que a transparência pública traz novas perspectivas para a "public accountability", por passar a verificar se as informações estão sendo acessíveis aos que se destinam (cidadãos e órgãos fiscalizadores).

Alguns estudos anteriores abordam a accountability no âmbito público no Brasil, quais sejam: Campos (1990), como sendo um dos primeiros registros de estudos publicados no Brasil sobre accountability no setor público, sendo o foco do estudo a divulgação da accountability no cenário brasileiro; Akutsu e Pinho (2002), realizaram um estudo para analisar como a internet foi utilizada pelos gestores públicos para incrementar a accountability através de um estudo de caso em 20 portais das três esferas de governo; Platt Neto, Cruz, Ensslin e Ensslin (2007), efetuaram uma revisão da literatura acerca da importância da disponibilização da informação dos gastos públicos, por meios eletrônicos (devido a determinação legal), favorecendo o exercício da cidadania pela população.

Ainda sobre estudos anteriores: Keunecke et al. (2011), observaram o nível de transparência dos gastos públicos nos cinco municípios mais populosos de Santa Catarina; Raupp e Pinho
(2013), realizaram um estudo nos endereços eletrônicos das Câmaras Municipais de Santa Catarina nos municípios abaixo de 10 mil habitantes para verificar o cumprimento da transparência das contas públicas, pesquisando 93 endereços eletrônicos; e Leite Filho, Colares e Andrade (2015), efetuaram uma pesquisa para averiguar se os maiores municípios mineiros cumprem a LC $n^{\circ}$ 131/209, disponibilizando as informações da execução orçamentária e financeira do município, em tempo real, descobrindo a condição de transparência da gestão fiscal pública municipal em tais municípios.

No contexto apresentado, torna-se oportuno verificar o nível de accountability nos municípios brasileiros, para tanto, emerge a seguinte questão de pesquisa: Qual o nível de accountability nos municípios brasileiros da Região Sul do Brasil? Para responder à tal questão tem-se como objetivo verificar o nível de accountability nos municípios da Região Sul do Brasil.

Justifica-se o presente trabalho pela evidenciação de Nakagawa, Relvas e Dias Filho (2007) ao destacarem que accountability trata-se de um tema incipiente em pesquisas, encontrado em desenvolvimento no Brasil. Keunecke et al. (2011) recomendam a replicação e disseminação da metodologia utilizada em seu estudo, visando identificar o cumprimento da "Lei de Transparência", identificando pontos de melhorias para um efetivo controle da gestão dos recursos públicos.

Cabe salientar que a pesquisa se torna relevante por contribuir com o tema em desenvolvimento no setor público; por ampliar a amostra utilizada nos estudos anteriores, visando uma maior evidenciação do nível e verificação dos determinantes de accountability nos municípios da Região Sul do Brasil; e por contribuir para a sociedade com informações acerca do cumprimento da legislação brasileira.

\section{REFERENCIAL TEÓRICO}

\subsection{New Public Management}

A New Public Management (NPM) teve início a partir dos anos 80 (destaque-se o Reino Unido, Nova Zelândia, Austrália e os países escandinavos) e nos anos 90 estendeu-se para os Estados Unidos da América e para o Brasil (Coutinho, 2000). Hood (1995) destaca como preceito base da NPM a diminuição/remoção de diferenças entre o setor público e o setor privado, alterando a ênfase de prestação de contas para um processo mais amplo, visando a evidenciação de resultados auferidos na gestão dos recursos públicos.

Segundo Hood (1995), pesquisadores têm associado a NPM com sete dimensões, sendo que dentre essas destacam-se quatro mudanças atreladas à eficiência e à prestação de contas, quais sejam: direcionamento à maior utilização de práticas de gestão que são utilizados no setor privado; maior ênfase na economia e disciplina na utilização de recursos públicos, buscando outras alternativas e formas menos onerosas de fornecer os serviços públicos; controle mais efetivo por gestores do topo, envolvendo regras de gestão pessoal para evitar o favorecimento pessoal em detrimento do social; e mudança para padrões mais explícitos e mensuráveis de desempenho organizacional, tanto em escala, nível 
e conteúdo dos serviços prestados em relação a padrões profissionais e experiências setoriais.

Quanto a NPM, Pereira (1996) destaca algumas características, como a descentralização política, observada pela transferência de recursos e atribuições políticas para governos regionais e locais (descentralização); organizações mais enxutas com poucos níveis hierárquicos; desígnio da confiança limitada em detrimento da total desconfiança; controle a posteriori, executado através da análise dos resultados dos processos administrativos; e a organização pública destinada ao atendimento do cidadão, visando o coletivo e não a interesses de minorias.

Nesse sentido, nota-se que a tendência da NPM tem sido caracterizada em algumas dimensões, como parte da reestruturação dos serviços públicos, caracterizados por casos de descentralização e privatização; mudança do estilo de gestão da administração pública com foco principal na gestão eficiente dos serviços públicos; esforços para demonstrar os ganhos de eficiência e os níveis de desempenho; e a responsabilização dos responsáveis pela gestão dos recursos públicos (Lapsley, 1999).

Cabe destacar que na NPM a contabilidade surge como elemento principal na nova concepção de prestação de contas, ofertando dados e métodos para análise e demonstração da aplicação dos recursos (Hood, 1995). Lapsley (1999) reforça orientando que a NPM oferece um papel fundamental para a contabilidade, visto que dela são extraídos dados para a elaboração de análises de medidas de desempenho.

A NPM tem forjado mudanças no pensamento, não só na contabilidade, mas em todas as áreas da gestão pública, fazendo com que a cautela e conservadorismo abram espaço para flexibilidade e para iniciativas de controles gerenciais, fato que tem demandado dos gestores uma visão focada no desempenho, no cidadão (Coutinho, 2000) e em abordagens proativas (Packer \& Gould, 1999)

No contexto da administração voltada ao cidadão, Coutinho (2000) afirma que os avanços tecnológicos têm auxiliado nesse processo, através de softwares, redes de compartilhamento de dados internos e externos. Nesse sentido, destaca-se a utilização da internet (através de sites e portais de transparência) para disponibilização de dados a população integrando o governo com a sociedade (Coutinho, 2000). O autor ainda disponibiliza uma lista (não exaustiva) com orientação para a implantação de uma administração pública voltada ao cidadão, como segue na tabela abaixo:

Tabela 1 -Orientação para implantação de administração pública voltada para o cidadão

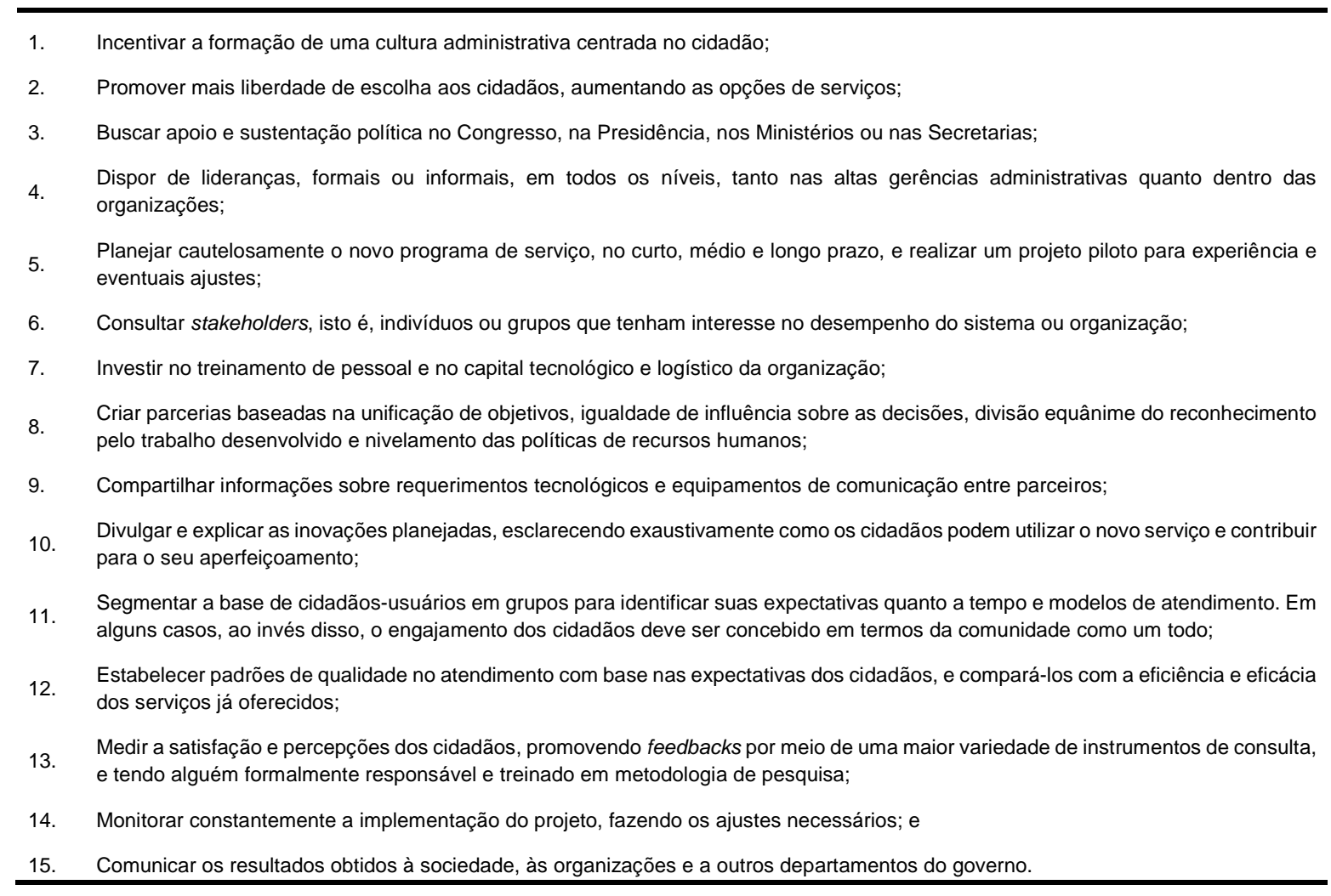

Fontes: Adaptado de Coutinho (2000).

Nesse sentido de implantação da NPM, Pollitt, Van Thiel e Homburg (2007) e Perci, Pieranti e Rodrigues (2008) ressaltam que há diferenças entre a NPM implantada em diversos países, pois a NPM tem que ser adaptada para a realidade cultural, social e política local. Os autores ainda destacam que a possibilidade de adaptação a situações e características específicas da administração pública se dá pela orientação da NPM à flexibilidade e aos processos inovadores.

Perci et al. (2008), enfatizam que um novo modelo de gestão pública possui processos próprios e diferentes da gestão pública tradicional, ou seja, as redes de governança modificam-se significativamente com a interação entre governo e a sociedade; e 
através da disponibilização de serviços por meio do setor privado ou do terceiro setor. Ao adaptar-se às características locais, devese observar a NPM como "um modelo normativo pós-burocrático para a estruturação e a gestão da administração pública baseado em valores de eficiência, eficácia e competitividade" (Secchi, 2009, p. 354).

Como há um crescente interesse dos governos e da sociedade para o alcance de melhores resultados no desempenho público (Pérez-López et al. 2015), torna-se salutar a constante avaliação do desempenho gestão pública (visando a eficiência e a eficácia) e à accountability (Ramos \& Schabbach, 2012), visando fomentar a divulgação dos resultados e o controle social através dos sítios eletrônicos. Tal tema será abordado no próximo tópico.

\subsection{Accountability}

O surgimento da accountability pode estar relacionado à Revolução Industrial associado ao início do capitalismo, visto a necessidade de controle e responsabilização dos gestores pela utilização dos recursos (Pinho \& Sacramento, 2009). A expressão accountability aparece no cenário brasileiro no final do século $X X$, mesmo sendo recorrente na Inglaterra desde o final do século XVIII. Assim, em 1975, Anna Maria Campos inicia a pesquisa sobre a accountability, após ouvir pela primeira vez o termo em uma aula do curso de pós-graduação em administração pública nos Estado Unidos da América (EUA).

Já em 1990, teve-se a primeira publicação no Brasil utilizando o termo accountability, com a publicação do artigo "Accountability: quando poderemos traduzi-la para o português? " na Revista de Administração Pública (RAP). Nesta publicação, Campos (1990) destaca que o conceito accountability ainda não foi compreendido pelos profissionais da administração pública e que não se encontra uma tradução literal para o português, fazendo com que o seu uso seja feito no idioma inglês, sendo, muitas vezes, o fator da não compreensão por aqueles que o utilizam. Neste sentido, Pinho e Sacramento (2009), identificam que não há uma palavra em português para traduzir a expressão accountability e sim traduções diferentes, originando termos que convergem.

No intuito de ampliar o entendimento do termo Pinho e Sacramento (2009) buscaram o significado em dicionários na linha inglesa, e destacam que a origem da palavra é antiga e consta nos dicionários ingleses desde 1794 , mas não são todos que apresentam a palavra accountability, entretanto, naqueles que a contemplava identificou-se o significado como responsabilização e prestação de contas. Posteriormente os autores realizaram buscas nos dicionários de inglês-português com o objetivo de verificar possíveis traduções para o termo accountable, o qual foi o mais próximo encontrado por eles de accountability. Nessas buscas os autores identificaram como possíveis traduções as palavras "responsável" e "prestar contas".

Com a intenção de ampliar o entendimento do conceito, Nakagawa et al. (2007) ressaltam após múltiplos estudos que a palavra "confiabilidade" como a forma mais adequada de traduzir a expressão accountability. Entretanto por questões culturais e acadêmicas os autores mantêm a utilização do termo em inglês em seus estudos.

Pinho e Sacramento (2009), preocupados com o contexto em que essa expressão surge, qual seja, a Revolução Industrial e o início do capitalismo, ainda comentam que o termo accountability não deveria ser traduzido para o português em virtude da conjuntura histórica da administração pública brasileira. Os autores acrescentam que a palavra accountability está sendo utilizada no Brasil para expressar, de forma implícita, a responsabilização pessoal pelos atos praticados e, de forma explícita, a prestação de contas e transparência na esfera pública ou privada.

Nesse contexto, Raupp e Pinho (2013) afirmam que a expressão accountability demanda a disponibilização, pelo poder público, das informações de seu desempenho e dos resultados das análises de desempenho e gestão dos recursos públicos. Os autores ainda ressaltam que a prestação de contas não pode limitar-se ao cumprimento das leis de Responsabilidade Fiscal, Lei da Transparência e a Lei de Acesso a Informações Públicas, mas deve abranger uma análise avançada sobre a disponibilização de informações das contas públicas. Raupp e Pinho (2013) ainda mencionam que, os relatórios precisam ser apresentados em conjunto e de forma simplificada para a compreensão por todos os cidadãos.

Rocha (2013) destaca o que o conceito accountability é uma expressão estrangeira inserida na administração pública brasileira que não absorve seu conceito na totalidade, pois a tradução como "prestação de contas" expressa parte do todo. Além da prestação de contas deve-se considerar a sua concretização por meio do cidadão no momento que este exerce a escolha dos seus representantes no governo; e, em caráter mais específico, a partir da atuação da imprensa, das organizações e associações de sociedade civil e dos cidadãos por meio de fiscalizações nas diferentes estruturas de governo (Rocha, 2013). Rocha (2013) complementa que existem dois planos diferentes para accountability, suportado por O'Donnell (1998) ao realizar a diferenciação de accountability de forma vertical e horizontal. A forma vertical é fruto da ação política do cidadão e a horizontal ocorre a partir do Estado, por meio dos órgãos e agências estatais (O'Donnell,1998; Rocha, 2013).

Campos (1990) destaca que a obrigação que está inserida no termo accountability expressa à responsabilidade do indivíduo diante de outro, traduzindo o que o indivíduo portador de cargo público necessita proporcionar. Neste sentido Rocha (2013) e Schillemans (2015) corrobora afirmando que accountability é uma forma de avaliar e responsabilizar os indivíduos portadores de cargos públicos pelo uso do poder a eles conferido pelos cidadãos, além de intensificar a interação entre os cidadãos e os gestores.

Nesse contexto fica evidente que o termo accountability traduzido para o português como "prestação de contas" e "responsável" não corresponde à totalidade da conceituação aplicada ao contexto brasileiro. Assim, ressalta-se a necessidade da accountability por meio do acompanhamento e fiscalização dos atos gerados pelos agentes públicos. Deste modo, este estudo posiciona accountability, em sentido restrito, como transparência 
dos atos e fatos governamentais disponibilizados na rede mundial de computadores.

\subsection{Estudos Anteriores}

Alguns autores têm despendido esforços para estudo e entendimento do tema accountability, analisando acerca do cumprimento da legislação brasileira, a forma como estão sendo disponibilizados os dados para prestação de contas e o nível transparência dos dados e as informações da gestão dos recursos públicos (Akutsu \& Pinho, 2002; Cruz, Silva \& Santos, 2009; Keunecke, Teles \& Flach, 2011; Leite Filho, Colares \& Andrade, 2015; Platt Neto et al., 2007; Raupp \& Pinho, 2013; Sacramento \& Pinho, 2007).

Akutsu e Pinho (2002) realizaram um estudo para analisar como a internet foi utilizada pelos gestores públicos para incrementar a accountability através de um estudo de caso em 20 portais das três esferas de governo. Foram analisados os portais dos governos federal, do Distrito Federal, dos Estados de São Paulo, Rio de Janeiro, Minas Gerais, Rio Grande do Sul, Paraná, Bahia, Santa Catarina, Pernambuco e Ceará, e dos municípios de São Paulo, Rio de Janeiro, Belo Horizonte, Porto Alegre, Curitiba, Salvador, Florianópolis, Recife e Fortaleza. Os achados do estudo evidenciaram a ausência de accountability na maior parte dos casos. Alguns resultados evidenciam avanço dos gestores, buscando uma maior accountability e a constituição de uma sociedade mais democrática.

Sacramento e Pinho (2007) desenvolveram um estudo para investigar os níveis de transparência da gestão fiscal pública de informações municipais, com evidência na participação popular por meio da Lei de Responsabilidade Fiscal em seis municípios da região metropolitana de Salvador no ano de 2002. Os resultados assinalaram que os níveis de transparência da gestão fiscal pública de informações municipais são pouco desenvolvidos e apontam que ainda é necessário muito trabalho para que a transparência se torne realidade nos municípios analisados.

Platt Neto et al., (2007), realizaram um estudo para descrever a obrigação e a abrangência dos princípios da publicidade e da transparência na administração pública brasileira, na divulgação das contas públicas. Para cumprir o objetivo do estudo foi realizada uma revisão da literatura da importância de disponibilizar as informações dos gastos públicos por meios eletrônicos. O estudo apontou a legalidade, a abrangência dos princípios da publicidade e da transparência aplicados na evidenciação das contas do setor público. Os autores identificaram também que a partir da Lei de Responsabilidade Fiscal e da Lei n 9.755/98 a internet tornou-se o instrumento mais aprimorado para a divulgação das contas públicas. Os resultados do estudo apontaram que a transparência na administração pública, garante o atendimento das normas legais e favorece o exercício da cidadania pela população.

Cruz, Silva e Santos (2009) realizaram um estudo para examinar o nível de transparência da gestão fiscal pública por meio da internet e os fatores que influenciam na evidenciação nos maiores municípios do Estado do Rio de Janeiro. Os resultados da pesquisa evidenciam que os municípios em estudo exibiram baixos níveis de transparência da gestão fiscal pública, sendo incompatível com o desenvolvimento econômico-social. Os autores destacam que os maiores níveis de transparência da gestão fiscal pública eletrônica ocorreram nos maiores municípios com os melhores indicadores socioeconômicos.

Keunecke et al. (2011), desenvolveram um estudo para observar o nível de transparência dos gastos públicos nos cinco municípios mais populosos de Santa Catarina identificando se os municípios estão atendendo as obrigações da Lei de Responsabilidade Fiscal. O instrumento utilizado nesta pesquisa foi o desenvolvido por Biderman e Puttomatti (2011), o qual foi atribuído um índice de transparência aos municípios estudados, resultando em um ranking. Os achados da pesquisa identificaram que os municípios analisados cumprem a Lei Complementar nำ131 de 2009 e destaca a importância da divulgação das contas públicas para promover a compreensão da accountability por todos os cidadãos.

Raupp e Pinho (2013) realizaram um estudo nos endereços eletrônicos das Câmaras Municipais de Santa Catarina nos municípios abaixo de 10 mil habitantes para verificar o cumprimento da transparência das contas públicas, pesquisando 93 endereços eletrônicos e propondo um ranking com relação à accountability. $A$ construção do ranking foi baseada no grau decrescente de condições de construção das três dimensões analisadas: prestação de contas, transparência e participação. Os achados da pesquisa demonstram evidências de que os portais eletrônicos não são utilizados como tecnologia de promoção da accountability, com algumas exceções. Foi possível verificar por meio do ranking construído que há uma ausência de construção de accountability, os portais eletrônicos de câmaras municipais do Estado de Santa Catarina são apenas murais eletrônicos.

Leite Filho et al. (2015), efetuaram uma pesquisa para averiguar se os maiores municípios mineiros cumprem a LC nำ 131/209 disponibilizando as informações da execução orçamentária e financeira do município, em tempo real, descobrindo a condição de transparência da gestão fiscal pública municipal nestes municípios. Os resultados da pesquisa evidenciaram que houve variação nos níveis de transparência da gestão pública municipal e nos níveis de transparência da gestão fiscal pública municipal nos municípios em análise, visto que o nível de transparência da gestão fiscal pública municipal estava relacionado com o tamanho da população e com o desenvolvimento econômico e social dos municípios.

\section{PROCEDIMENTOS METODOLÓGICOS}

O enquadramento da pesquisa quanto ao objetivo da pesquisa classifica-se como exploratória, abordagem qualitativa e multicaso (Klein, Silva, Machado \& Azevedo, 2015). Quanto a coleta de dados, foram analisados os dados disponíveis nos sites das prefeituras que compõe a amostra. Para identificar o nível de transparência foi utilizada uma adaptação da metodologia de Keunecke et al. (2011) e Speck (2014), conforme demonstra-se na tabela abaixo. 
Tabela 2- Medição do Nível de Transparência

\begin{tabular}{|c|c|}
\hline Categoria: Conteúdo & Pontos Max. \\
\hline Etapas da Execução da Despesa Orçamentária & 8 \\
\hline Etapas da Execução da Receita Orçamentária & 4 \\
\hline Leis e Relatórios Orçamentários & 5 \\
\hline Patrimônio & 3 \\
\hline Outros Poderes & 4 \\
\hline Subtotal & 24 \\
\hline Categoria: Série Histórica e Frequência de Atualização & Pontos Max. \\
\hline Série Histórica & 4 \\
\hline Atualização & 6 \\
\hline Subtotal & 10 \\
\hline Categoria: Usabilidade & Pontos Max. \\
\hline Possibilidade de Download & 2 \\
\hline Delimitação Temporal da Consulta & 5 \\
\hline Formulário de Consulta & 1 \\
\hline Liberdade de Preenchimento do Formulário Principal & 3 \\
\hline Gráficos & 2 \\
\hline Subtotal & 13 \\
\hline Total Geral & 47 \\
\hline
\end{tabular}

Fontes: Adaptado de Keunecke et al. (2011) e Speck (2014).

Observa-se na Tabela 2, os indicadores e a pontuação para a quantificação do nível de transparência dos municípios. Para que não tenha viés dos autores desta pesquisa, os pontos das Categorias Conteúdo e Usabilidade são somados de acordo com a disponibilização dos dados, ou seja, cada ponto refere-se a um conteúdo disponibilizado (Por exemplo: a Etapa da Execução da Despesa Orçamentária possui 8 divisões, sendo cada uma valendo um ponto para cada item, a Possibilidade de Download possui 2 divisões, sendo que cada uma vale 1 ponto, e o mesmo é adotado para as Categorias e as suas subdivisões).

Já a Categoria Série Histórica e Frequência de Atualização a pontuação é diferente das outras categorias. Na Série Histórica tem-se a pontuação de 1 ponto para disponibilização de 1 a 3 anos, 2 pontos para 4 a 6 anos, 3 pontos para 7 a 9 anos e 4 pontos para 10 anos para cima, de forma não cumulativa e a Atualização, da mesma forma, concede os pontos de acordo com o lapso de disponibilização dos dados, como segue: 6 pontos para atualização online (24h), 5 pontos de 2 a 7 dias, 4 pontos de 8 a 15 dias, 3 pontos de 16 a 30 dias, 2 pontos de 31 a 60 dias e 1 ponto a partir de 60 dias para disponibilização.

O cálculo do índice de transparência se dá pelo somatório das pontuações dos três subtotais (Conteúdo, Série Histórica e Frequência de Atualização e Usabilidade). Observa-se então que:

$\mathrm{C}=$ Conteúdo tem a pontuação máxima de 24 pontos;

$\mathrm{SH}=$ Série Histórica e Frequência de Atualização tem a pontuação máxima de 10 pontos; e

$U=$ Usabilidade tem a pontuação máxima de 13 pontos.

Assim, Cmá $x+$ SHmáx + Umáx $=47$ pontos .

A coleta dos dados ocorreu entre os meses de novembro e dezembro de 2015 na amostra não probabilística de 60 municípios da Região Sul do Brasil, segregando entre os 10 municípios mais populosos e 10 municípios menos populosos dos Estados do Paraná, Santa Catarina e Rio Grande do Sul, segundo dados do IBGE (2010). A tabela a seguir demonstra a amostra utilizada na presente pesquisa.

Tabela 3- Amostra utilizada na pesquisa

\begin{tabular}{|c|c|c|}
\hline Estado & $\begin{array}{l}\text { Mais/Menos } \\
\text { Populoso }\end{array}$ & Municípios \\
\hline \multirow{2}{*}{ Paraná } & Mais populosos & $\begin{array}{l}\text { Curitiba, Londrina, Maringá, Ponta Grossa, Cascavel, São José do Pinhais, Foz do lguaçu, Colombo, Guarapuava e } \\
\text { Paranaguá. }\end{array}$ \\
\hline & Menos populosos & $\begin{array}{l}\text { Santo Antônio do Paraíso, Mirador, Iguaçu, Guaporema, São Manoel do Paraná, Esperança Nova, Miraselva, Santa } \\
\text { Inês, Nova Aliança do Ivaí e Jardim Olinda. }\end{array}$ \\
\hline \multirow{2}{*}{ Santa Catarina } & Mais populosos & Joinville, Florianópolis, Blumenau, São José, Criciúma, Chapecó, Itajaí, Lages, Jaraguá do Sul e Palhoça. \\
\hline & Menos populosos & $\begin{array}{l}\text { Cunhataí, Barra Bonita, Macieira, Jardinópolis, Paial, Tigrinhos, Presidente Castello Branco, Flor do Sertão, Lajeado } \\
\text { Grande e Santiago do Sul. }\end{array}$ \\
\hline \multirow{2}{*}{ Rio Grande do Sul } & Mais populosos & $\begin{array}{l}\text { Porto Alegre, Caxias do Sul, Pelotas, Canoas, Santa Maria, Gravataí, Viamão, Novo Hamburgo, São Leopoldo e Rio } \\
\text { Grande. }\end{array}$ \\
\hline & Menos populosos & $\begin{array}{l}\text { Carlos Gomes, Guabiju, Lagoa dos Três Cantos, Tupanci do Sul, Vista Alegre do Prata, Montauri, Coqueiro Baixo, } \\
\text { Engenho Velho, União da Serra, André da Rocha. }\end{array}$ \\
\hline
\end{tabular}

Fontes: dados da pesquisa.

Visando coesão na análise dos dados, entende-se como maiores municípios os mais populosos e como menores os menos populosos. Após a coleta de dados no site dos municípios explicitados na Tabela 3, os dados serão organizados em uma planilha eletrônica, e após, será elaborado um ranking dos municípios a fim de evidenciar o nível de accountability. Posteriormente, visando uma análise aprofundada dos dados, verificar-se-á a correlação existente entre o Ranking do nível de Accountability com o Ranking do Desempenho Socioeconômico e do Desempenho Econômico-Financeiro dos municípios analisados.

Para tanto, utilizou-se a Correlação de Kendall tendo em vista a relação não linear entre os indicadores e a característica ordinal da escala de classificação do Ranking (Hair, Babin, Money \& 
Samouel, 2005). Para o cálculo da Correlação de Kendall utilizouse o software estatístico IBM SPSS Statistics $22{ }^{\circledR}$.

Para elaboração do Ranking do Desempenho Socioeconômico e do Ranking do Desempenho Econômico-financeiro utilizou-se os indicadores e quocientes, respectivamente, conforme explicitado na Tabela 4, disposta abaixo, sendo que os indicadores
Socioeconômicos foram coletados no site do Instituto Brasileiro de Geografia e Estatística (IBGE) e no site Atlas do Desenvolvimento Humano no Brasil; e os quocientes de Desempenho Econômicofinanceiro foram calculados a partir dos Balanços Patrimoniais disponibilizados no site dos municípios analisados.

Tabela 4 - Indicadores socioeconômicas e Quocientes de desempenho econômico-financeiro

\begin{tabular}{|c|c|c|c|}
\hline \multicolumn{4}{|c|}{ INDICADORES } \\
\hline \multirow{6}{*}{ 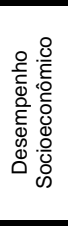 } & \multirow{4}{*}{$\begin{array}{l}\text { Índice de Desenvolvimento } \\
\text { Humano Municipal }\end{array}$} & \multicolumn{2}{|c|}{ IDH-M } \\
\hline & & \multicolumn{2}{|c|}{ IDH-M Renda } \\
\hline & & \multicolumn{2}{|c|}{ IDH-M Longevidade } \\
\hline & & \multicolumn{2}{|c|}{ IDH-M Educação } \\
\hline & \multirow{2}{*}{$\begin{array}{l}\text { Ocupação da população de } \\
18 \text { anos ou mais }\end{array}$} & \multirow{2}{*}{\multicolumn{2}{|c|}{$\begin{array}{c}\text { Taxa de Atividade } \\
\text { Grau de Formalização dos Ocupados }-18 \text { anos ou mais }\end{array}$}} \\
\hline & & & Grau de Formalização dos Ocupados - 18 anos ou mais \\
\hline \multicolumn{4}{|c|}{ QUOCIENTES } \\
\hline \multirow{5}{*}{ 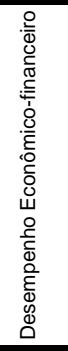 } & \multirow{5}{*}{ Balanço Patrimonial } & Liquidez Imediata & $\frac{\text { Disponibilidades }}{\text { Passivo Circulante }}$ \\
\hline & & Liquidez Corrente & $\frac{\text { Ativo Circulante }(A C)}{\text { Passivo Circulante }(P C)}$ \\
\hline & & Liquidez Seca & $\frac{\text { Disp. }+ \text { Crédito a Curto Prazo }}{\text { Passivo Circulante }}$ \\
\hline & & Liquidez Geral & $\frac{A C+\text { Realiz. Longo Prazo }}{P C+P \tilde{\mathrm{n}} C}$ \\
\hline & & Índice de Solvência & $\begin{array}{l}A C+\text { Ativo não Circulante }(A \tilde{n} C) \\
C+\text { Passivo não Circulante }(P \tilde{\mathrm{n}} C)\end{array}$ \\
\hline
\end{tabular}

Fontes: Adaptado de MCASP (2012), IBGE (2015) e PNUD (2015).

Após a coleta de dados nos sites dos municípios explicitados na Tabela 3, foi elaborado um ranking dos municípios, visando evidenciar o Desempenho Socioeconômico e Desempenho Econômico-financeiro. Para obtenção do Ranking dos Desempenhos analisados foram somado os resultados obtidos nos indicadores socioeconômicos e nos quocientes de desempenho econômico-financeiro. A análise dos indicadores e dos quocientes se dá por quanto maior o resultado obtido melhor será o desempenho da organização (Mcasp, 2012; Pnud, 2015).

\section{DISCUSSÃO E ANÁLISE DOS RESULTADOS}

Com a análise dos dados coletados e com o intuito de alcançar o objetivo do presente estudo, demonstra-se na Tabela 5 o Ranking de transparência, sendo que o primeiro é o município mais transparente e o último o menos transparente, contemplando toda a amostra da pesquisa.

Tabela 5 - Ranking de Transparência dos Municípios

\begin{tabular}{|c|c|c|c|c|c|c|c|}
\hline Posição & Estado & Municipio & Pontos & Posição & Estado & Município & Pontos \\
\hline 1 & SC & Joinville & 35 & 8 & RS & Carlos Gomes & 26 \\
\hline \multirow{3}{*}{2} & $\mathrm{SC}$ & Blumenau & 32 & 9 & $\mathrm{PR}$ & Ponta Grossa & 25 \\
\hline & $\mathrm{SC}$ & Itajaí & 32 & \multirow{5}{*}{10} & $\overline{P R}$ & Santa Inês & 24 \\
\hline & $\overline{P R}$ & Paranaguá & 32 & & RS & Canoas & 24 \\
\hline \multirow{5}{*}{3} & $\mathrm{SC}$ & Chapecó & 31 & & $\mathrm{RS}$ & São Leopoldo & 24 \\
\hline & $\mathrm{SC}$ & Tigrinhos & 31 & & RS & Rio Grande & 24 \\
\hline & $\mathrm{SC}$ & Santiago do Sul & 31 & & $\mathrm{RS}$ & Montauri & 24 \\
\hline & $\mathrm{PR}$ & Colombo & 31 & \multirow{5}{*}{11} & $P R$ & Cascavel & 23 \\
\hline & $\overline{R S}$ & Pelotas & 31 & & $\overline{P R}$ & Miraselva & 23 \\
\hline \multirow{3}{*}{4} & SC & Lages & 30 & & $\mathrm{RS}$ & Porto Alegre & 23 \\
\hline & $\mathrm{PR}$ & Santo Antônio do Paraíso & 30 & & RS & Engenho Velho & 23 \\
\hline & $\mathrm{PR}$ & Jardim Olinda & 30 & & $\mathrm{RS}$ & André da Rocha & 23 \\
\hline \multirow{3}{*}{5} & $\mathrm{SC}$ & Florianópolis & 29 & \multirow{2}{*}{12} & $\mathrm{SC}$ & Flor do Sertão & 22 \\
\hline & $\mathrm{RS}$ & Caxias do Sul & 29 & & $\mathrm{RS}$ & Tupanci do Sul & 22 \\
\hline & RS & Novo Hamburgo & 29 & \multirow{6}{*}{13} & $\overline{P R}$ & Mirador & 21 \\
\hline \multirow{6}{*}{6} & $\mathrm{SC}$ & Criciúma & 28 & & $\overline{P R}$ & Iguatu & 21 \\
\hline & $\mathrm{SC}$ & Jaraguá do Sul & 28 & & $\overline{P R}$ & Esperança Nova & 21 \\
\hline & $\mathrm{PR}$ & Guarapuava & 28 & & PR & Nova Aliança do Ivaí & 21 \\
\hline & $\mathrm{PR}$ & Guaporema & 28 & & $\mathrm{RS}$ & Santa Maria & 21 \\
\hline & $\mathrm{PR}$ & São Manoel do Paraná & 28 & & $\mathrm{RS}$ & Guabiju & 21 \\
\hline & RS & Lagoa dos Três Cantos & 28 & 14 & RS & Viamão & 19 \\
\hline \multirow{9}{*}{7} & $\mathrm{SC}$ & São José & 27 & 15 & $\overline{P R}$ & São José dos Pinhais & 18 \\
\hline & $\mathrm{SC}$ & Palhoça & 27 & 16 & $\mathrm{RS}$ & União da Serra & 16 \\
\hline & $\overline{P R}$ & Curitiba & 27 & 17 & $\mathrm{SC}$ & Barra Bonita & 13 \\
\hline & $\mathrm{PR}$ & Londrina & 27 & \multirow{3}{*}{18} & $\mathrm{SC}$ & Cunhataí & 9 \\
\hline & $\mathrm{PR}$ & Maringá & 27 & & $\mathrm{SC}$ & Presidente Castello Branco & 9 \\
\hline & $\mathrm{PR}$ & Foz do Iguaçu & 27 & & $\mathrm{SC}$ & Lajeado Grande & 9 \\
\hline & $\mathrm{RS}$ & Gravataí & 27 & 19 & $\mathrm{SC}$ & Paial & 8 \\
\hline & $\mathrm{RS}$ & Vista Alegre do Prata & 27 & \multirow{2}{*}{20} & $\mathrm{SC}$ & Macieira & 7 \\
\hline & $\mathrm{RS}$ & Coqueiro Baixo & 27 & & $\mathrm{SC}$ & Jardinópolis & 7 \\
\hline
\end{tabular}

Fonte: Dados da pesquisa.

Observando a Tabela 5, verifica-se que o município mais transparente é Joinville por alcançar o total de 35 pontos, seguido por Blumenau, Itajaí e Paranaguá empatados na segunda posição com 31 pontos. Destaca-se o Estado de Santa Catarina por possuir $75 \%$ dos municípios com maior nível de transparência, seguido por Paraná com $25 \%$ e Rio Grande do Sul com $0 \%$. 
No final, a direita da Tabela 5, evidencia-se os municípios com menor nível de transparência, sendo que todos os municípios que compõe as três últimas posições pertencem ao estado de Santa Catarina. Os municípios são Cunhataí, Presidente Castello Branco e Lajeado Grande com 09 pontos; Paial com 08 pontos; e Macieira e Jardinópolis com 07 pontos.
Nesse contexto, os achados corroboram com Akutsu e Pinho (2002) evidenciando que alguns gestores estão buscando uma maior accountability, cabe salientar que a amostra, o período analisado e a metodologia utilizada pelo estudo anterior diferem da presente pesquisa.

A próxima tabela demonstra o Ranking dos maiores municípios dos Estados da Região Sul do Brasil.

Tabela 6 - Ranking de Transparência dos Maiores Municípios

\begin{tabular}{|c|c|c|c|c|c|c|c|}
\hline Posição & Estado & Município & Pontos & Posição & Estado & Município & Pontos \\
\hline 1 & $\mathrm{SC}$ & Joinville & 35 & \multirow{6}{*}{7} & $\mathrm{SC}$ & Palhoça & 27 \\
\hline \multirow{3}{*}{2} & SC & Blumenau & 32 & & PR & Curitiba & 27 \\
\hline & SC & Itajaí & 32 & & PR & Londrina & 27 \\
\hline & $\mathrm{PR}$ & Paranaguá & 32 & & PR & Maringá & 27 \\
\hline \multirow{3}{*}{3} & $\mathrm{SC}$ & Chapecó & 31 & & PR & Foz do Iguaçu & 27 \\
\hline & PR & Colombo & 31 & & $\mathrm{RS}$ & Gravataí & 27 \\
\hline & RS & Pelotas & 31 & 8 & PR & Ponta Grossa & 25 \\
\hline 4 & SC & Lages & 30 & \multirow{3}{*}{9} & $\mathrm{RS}$ & Canoas & 24 \\
\hline \multirow{3}{*}{5} & SC & Florianópolis & 29 & & $\mathrm{RS}$ & São Leopoldo & 24 \\
\hline & $\mathrm{RS}$ & Caxias do Sul & 29 & & $\overline{\mathrm{RS}}$ & Rio Grande & 24 \\
\hline & $\overline{R S}$ & Novo Hamburgo & 29 & \multirow{2}{*}{10} & $\overline{\mathrm{PR}}$ & Cascavel & 23 \\
\hline \multirow{3}{*}{6} & $\overline{S C}$ & Criciúma & 28 & & $\overline{\mathrm{RS}}$ & Porto Alegre & 23 \\
\hline & $\overline{S C}$ & Jaraguá do Sul & 28 & 11 & $\overline{\mathrm{RS}}$ & Santa Maria & 21 \\
\hline & PR & Guarapuava & 28 & 12 & $\overline{\mathrm{RS}}$ & Viamão & 19 \\
\hline 7 & SC & São José & 27 & 13 & PR & São José dos Pinhais & 18 \\
\hline
\end{tabular}

Fonte: Dados da pesquisa.

A Tabela 6 demonstra os mesmos municípios da Tabela 5 na primeira posição, evidenciando que os municípios com maior nível de accountability estão entre os mais populosos, já nas últimas posição tem-se uma mudança. As últimas posições comportam dois municípios do Rio Grande do Sul (Santa Maria, com 21 pontos e Viamão, com 19 pontos) e o município paranaense São José dos Pinhais, com 18 pontos.
Os resultados corroboram com Keunecke et al. (2011), apesar da amostra e o período serem diferentes, pode-se inferir que os cinco municípios mais populosos de Santa Catarina (Joinville e Blumenau) promovem maior divulgação das contas públicas.

$\mathrm{Na}$ próxima tabela demonstra-se o Ranking dos menores municípios dos Estados da Região Sul do Brasil.

Tabela 7- Ranking de Transparência dos Menores Municípios

\begin{tabular}{|c|c|c|c|c|c|c|c|}
\hline Posição & Estado & Município & Pontos & Posição & Estado & Município & Pontos \\
\hline \multirow{2}{*}{1} & SC & Tigrinhos & 31 & \multirow{2}{*}{8} & SC & Flor do Sertão & 22 \\
\hline & SC & Santiago do Sul & 31 & & RS & Tupanci do Sul & 22 \\
\hline \multirow{2}{*}{2} & $\mathrm{PR}$ & Santo Antônio do Paraíso & 30 & \multirow{5}{*}{9} & $\mathrm{PR}$ & Mirador & 21 \\
\hline & PR & Jardim Olinda & 30 & & $\mathrm{PR}$ & Iguatu & 21 \\
\hline \multirow{3}{*}{3} & PR & Guaporema & 28 & & PR & Esperança Nova & 21 \\
\hline & PR & São Manoel do Paraná & 28 & & PR & Nova Aliança do Ivaí & 21 \\
\hline & RS & Lagoa dos Três Cantos & 28 & & RS & Guabiju & 21 \\
\hline \multirow{2}{*}{4} & RS & Vista Alegre do Prata & 27 & 10 & RS & União da Serra & 16 \\
\hline & RS & Coqueiro Baixo & 27 & 11 & SC & Barra Bonita & 13 \\
\hline 5 & RS & Carlos Gomes & 26 & \multirow{3}{*}{12} & SC & Cunhataí & 9 \\
\hline \multirow{2}{*}{6} & $\mathrm{PR}$ & Santa Inês & 24 & & SC & Presidente Castello Branco & 9 \\
\hline & RS & Montauri & 24 & & SC & Lajeado Grande & 9 \\
\hline \multirow{3}{*}{7} & PR & Miraselva & 23 & 13 & SC & Paial & 8 \\
\hline & RS & Engenho Velho & 23 & \multirow{2}{*}{14} & SC & Macieira & 7 \\
\hline & RS & André da Rocha & 23 & & SC & Jardinópolis & 7 \\
\hline
\end{tabular}

Fonte: Dados da pesquisa.

$\mathrm{Na}$ Tabela 7, tem-se que as duas primeiras posições são divididas entre Santa Catarina e Paraná, sendo que Santa Catarina fica com a primeira posição, com 31 pontos (Tigrinhos e Santiago do Sul) e o Paraná com a segunda posição, com 30 pontos (Santo Antônio do Paraíso e Jardim Olinda). As últimas posições nada diferem da Tabela 5.

Comparando a Tabela 6 e 7, observa-se que na variação (diferença entre os primeiros e último colocados) no nível de transparência dos menores municípios é de 24 pontos e entre os maiores municípios é de 17 pontos. Assim, tem-se maior disparidade do nível de transparência nos municípios menores que nos maiores.

A próxima tabela evidencia o total de pontos conquistados pelos Estados da Região Sul do Brasil segregando entre os maiores e os menores municípios.

\begin{tabular}{|c|c|c|c|}
\hline Posição & Estado & Descrição & Pontos \\
\hline \multirow{3}{*}{1} & \multirow{3}{*}{ PR } & Maiores & 265 \\
\hline & & Menores & 247 \\
\hline & & Total: & 512 \\
\hline \multirow{3}{*}{2} & \multirow{3}{*}{ RS } & Maiores & 251 \\
\hline & & Menores & 237 \\
\hline & & Total: & 488 \\
\hline \multirow{3}{*}{3} & \multirow{3}{*}{ SC } & Maiores & 299 \\
\hline & & Menores & 146 \\
\hline & & Total: & 445 \\
\hline
\end{tabular}

A Tabela 8 demonstra que o Estado que mais pontuou foi o Paraná, obtendo o total de 512 pontos (com 35,43\% do total de pontos), seguido pelo Rio Grande do Sul, com 488 pontos (33,77\%) e Santa Catarina, com 445 pontos $(30,79 \%)$. Nesse sentido de 
análise, dentre os maiores municípios analisados tem-se o Estado de Santa Catarina em primeiro, com 299 pontos, seguido do Paraná, com 265 pontos, e do Rio Grade do Sul, com 251 pontos. Já dentre os menores municípios, a sequência é a mesma dos Estados que mais pontuaram, ou seja, Paraná, Rio Grande do Sul e Santa Catarina.
Após a apresentação do Ranking do nível de Accountability apresenta-se o Ranking de Desempenho Socioeconômico com o Ranking de Desempenho Financeiro dos municípios analisados, como segue:

Tabela 9 - Ranking de Desempenho Socioeconômico.

\begin{tabular}{|c|c|c|c|c|c|c|c|}
\hline Posição & Estado & Município & Pontos & Posição & Estado & Município & Pontos \\
\hline 1 & $\mathrm{SC}$ & Jaraguá do Sul & 167,23 & 31 & $\mathrm{RS}$ & Santa Maria & 139,34 \\
\hline 2 & SC & Blumenau & 163,66 & 32 & PR & Paranaguá & 138,69 \\
\hline 3 & SC & Chapecó & 160,11 & 33 & $\mathrm{PR}$ & Nova Aliança do Ivaí & 136,96 \\
\hline 4 & RS & Caxias do Sul & 160,06 & 34 & $\mathrm{PR}$ & Guarapuava & 134,78 \\
\hline 5 & SC & São José & 158,93 & 35 & $\mathrm{PR}$ & Foz do Iguaçu & 134,07 \\
\hline 6 & SC & Joinville & 156,98 & 36 & RS & Guabiju & 133,31 \\
\hline 7 & SC & Jardinópolis & 154,57 & 37 & $\mathrm{PR}$ & São Manoel do Paraná & 132,56 \\
\hline 8 & SC & Palhoça & 154,50 & 38 & RS & Pelotas & 131,70 \\
\hline 9 & SC & Itajaí & 153,69 & 39 & $\mathrm{PR}$ & Guaporema & 131,69 \\
\hline 10 & PR & São José dos Pinhais & 153,47 & 40 & RS & Rio Grande & 131,47 \\
\hline 11 & SC & Barra Bonita & 153,20 & 41 & $\mathrm{PR}$ & Santa Inês & 130,73 \\
\hline 12 & SC & Criciúma & 151,51 & 42 & $\mathrm{PR}$ & Jardim Olinda & 130,43 \\
\hline 13 & $\mathrm{PR}$ & Curitiba & 150,78 & 43 & $\mathrm{PR}$ & Mirador & 129,37 \\
\hline 14 & SC & Florianópolis & 150,63 & 44 & SC & Flor do Sertão & 127,91 \\
\hline 15 & SC & Cunhataí & 150,15 & 45 & RS & André da Rocha & 127,66 \\
\hline 16 & $\mathrm{PR}$ & Cascavel & 150,01 & 46 & RS & Vista Alegre do Prata & 127,63 \\
\hline 17 & $\mathrm{PR}$ & Colombo & 149,52 & 47 & SC & Presidente Castello Branco & 125,61 \\
\hline 18 & RS & Novo Hamburgo & 149,42 & 48 & SC & Tigrinhos & 125,49 \\
\hline 19 & $\mathrm{PR}$ & Maringá & 147,36 & 49 & SC & Macieira & 122,23 \\
\hline 20 & RS & São Leopoldo & 147,31 & 50 & $\mathrm{PR}$ & Esperança Nova & 121,50 \\
\hline 21 & RS & Canoas & 146,63 & 51 & RS & Lagoa dos Três Cantos & 120,50 \\
\hline 22 & RS & Porto Alegre & 145,95 & 52 & $\mathrm{PR}$ & Iguatu & 120,15 \\
\hline 23 & $\mathrm{PR}$ & Londrina & 145,02 & 53 & RS & União da Serra & 118,81 \\
\hline 24 & SC & Paial & 143,20 & 54 & $\mathrm{PR}$ & Santo Antônio do Paraíso & 117,36 \\
\hline 25 & RS & Gravataí & 142,73 & 55 & RS & Tupanci do Sul & 112,23 \\
\hline 26 & $\mathrm{PR}$ & Miraselva & 142,67 & 56 & SC & Santiago do Sul & 112,11 \\
\hline 27 & RS & Viamão & 142,53 & 57 & RS & Carlos Gomes & 110,71 \\
\hline 28 & $\mathrm{PR}$ & Ponta Grossa & 141,70 & 58 & RS & Montauri & 110,25 \\
\hline 29 & SC & Lages & 140,11 & 59 & RS & Engenho Velho & 106,04 \\
\hline 30 & SC & Lajeado Grande & 139,89 & 60 & RS & Coqueiro Baixo & 80,40 \\
\hline
\end{tabular}

Fonte: Dados da pesquisa

Observa-se na Tabela 9 que os municípios que possuem maior somatório dos indicadores Socioeconômicos são de Santa Catarina, os quais sejam: Jaraguá do Sul, Blumenau e Chapecó. Na outra ponta da tabela, os municípios que possuem menor somatório dos indicadores são do Rio Grande do Sul, são eles: Montauri, Engenho Velho e Coqueiro Baixo. Nota-se que o município de
Lajeado Grande (SC) fica posicionado no meio da tabela, ou seja, na $30^{2}$ posição, distanciado a 27,64 pontos da primeira posição e a 59,49 pontos da última posição.

Na próxima tabela demonstra-se o Ranking de Desempenho Econômico-financeiro calculado através dos resultados dos quocientes do Balanço Patrimonial, como segue:

Tabela 10- Ranking de Desempenho Econômico-financeiro

\begin{tabular}{|c|c|c|c|c|c|c|c|}
\hline Posição & Estado & Município & Pontos & Posição & Estado & Município & Pontos \\
\hline 1 & PR & Guaporema & $1.433,26$ & 28 & SC & Blumenau & 20,85 \\
\hline 2 & PR & Mirador & 265,41 & 29 & RS & Vista Alegre do Prata & 20,32 \\
\hline 3 & PR & Esperança Nova & 240,23 & 30 & $\mathrm{RS}$ & Pelotas & 18,31 \\
\hline 4 & PR & Maringá & 216,36 & 31 & SC & São José & 17,27 \\
\hline 5 & RS & Guabiju & 192,11 & 32 & SC & Paial & 16,99 \\
\hline 6 & PR & Santo Antônio do Paraíso & 180,53 & 33 & RS & Novo Hamburgo & 16,76 \\
\hline 7 & SC & Flor do Sertão & 144,20 & 34 & PR & Curitiba & 16,71 \\
\hline 8 & PR & Guarapuava & 116,78 & 35 & $\mathrm{RS}$ & Rio Grande & 15,83 \\
\hline 9 & RS & Montauri & 106,71 & 36 & SC & Jaraguá do Sul & 15,19 \\
\hline 10 & SC & Joinville & 90,79 & 37 & PR & Cascavel & 13,99 \\
\hline 11 & RS & União da Serra & 76,75 & 38 & PR & Miraselva & 12,63 \\
\hline 12 & $\mathrm{RS}$ & Viamão & 75,20 & 39 & SC & Presidente Castello Branco & 12,39 \\
\hline 13 & RS & Coqueiro Baixo & 66,49 & 40 & PR & Nova Aliança do Ivaí & 12,32 \\
\hline 14 & PR & São José dos Pinhais & 65,86 & 41 & $\mathrm{RS}$ & Canoas & 11,59 \\
\hline 15 & SC & Lajeado Grande & 57,64 & 42 & RS & Porto Alegre & 11,46 \\
\hline 16 & SC & Macieira & 53,70 & 43 & RS & Caxias do Sul & 11,12 \\
\hline 17 & PR & Ponta Grossa & 51,38 & 44 & RS & Engenho Velho & 10,90 \\
\hline 18 & SC & Cunhataí & 45,07 & 45 & PR & Santa Inês & 10,35 \\
\hline 19 & RS & Lagoa dos Três Cantos & 42,30 & 46 & PR & Jardim Olinda & 10,26 \\
\hline 20 & SC & Itajaí & 41,07 & 47 & SC & Chapecó & 9,94 \\
\hline 21 & PR & São Manoel do Paraná & 32,33 & 48 & SC & Jardinópolis & 9,91 \\
\hline 22 & PR & Paranaguá & 32,03 & 49 & SC & Criciúma & 9,17 \\
\hline 23 & PR & Londrina & 24,67 & 50 & PR & Foz do Iguaçu & 8,84 \\
\hline 24 & RS & Santa Maria & 24,30 & 51 & SC & Lages & 6,62 \\
\hline 25 & PR & Iguatu & 23,72 & 52 & $\mathrm{RS}$ & Gravataí & 5,07 \\
\hline 26 & RS & Carlos Gomes & 22,53 & 53 & RS & São Leopoldo & 4,78 \\
\hline 27 & SC & Tigrinhos & 20,91 & 54 & SC & Florianópolis & 4,05 \\
\hline
\end{tabular}

Fonte: Dados da pesquisa.

Verificando a Tabela 10, nota-se que seis municípios não foram contemplados, visto a falta de informações para o cálculo dos quocientes do Balanço Patrimonial, disponibilizados nos sites dos municípios analisados. Os municípios que ficaram de fora dessa 
análise foram: Palhoça, Barra Bonita e Santiago do Sul, de SC; Colombo, do PR; e Tupansi do Sul e André da Rocha, do RS.

Nesta tabela, os primeiros colocados são municípios do estado do Paraná, quais sejam: Guaporema, Mirador e Esperança Nova, diferenciando das posições demonstradas nos indicadores Socioeconômicos. O mesmo observa-se nas últimas posições, divididas entre SC e RS, sendo Lages/SC na $51^{\text {a }}$ posição, seguida de Gravataí/RS e São Leopoldo/RS e finalizando com Florianópolis/SC evidenciando o menor nível de Desempenho Econômico-financeiro dentre os municípios analisados.

Por fim, de posse dos Rankings de Desempenho Socioeconômico e Econômico-financeiro, calculou-se a Correlação de Kendall, conforme demonstra-se tabela a seguir:

Tabela 11 - Correlação de Kendall entre os Rankings

\begin{tabular}{|c|c|c|c|c|}
\hline \multicolumn{5}{|c|}{ Correlação entre Nível de Accountability com o Desempenho Socioeconômico } \\
\hline & & & Posição_Account & Posição_DS \\
\hline \multirow{6}{*}{ tau_b de Kendall } & \multirow{3}{*}{ Posição_Account } & Coeficiente de Correlação & 1,000 & $0,969^{* * *}$ \\
\hline & & Sig. (2 extremidades) & & 0,000 \\
\hline & & $\mathrm{N}$ & 60 & 60 \\
\hline & \multirow{3}{*}{ Posição_DS } & Coeficiente de Correlação & $0,969^{* * *}$ & 1,000 \\
\hline & & Sig. (2 extremidades) &, 000 & \\
\hline & & $\mathrm{N}$ & 60 & 60 \\
\hline \multicolumn{5}{|c|}{ Correlação entre Nível de Accountability com o Desempenho Econômico-Financeiro } \\
\hline \multirow{6}{*}{ tau_b de Kendall } & \multirow{3}{*}{ Posição_Account } & Coeficiente de Correlação & 1,000 & $0,969^{x+2 x}$ \\
\hline & & Sig. (2 extremidades) & & 0,000 \\
\hline & & $\mathrm{N}$ & 54 & 54 \\
\hline & \multirow{3}{*}{ Posição_DEF } & Coeficiente de Correlação & $0,969^{* * *}$ & 1,000 \\
\hline & & Sig. (2 extremidades) & 0,000 & \\
\hline & & $\mathrm{N}$ & 54 & 54 \\
\hline
\end{tabular}

***. A correlação é significativa no nível 0,01 (2 extremidades).

Legenda: Account = Accountability; DS = Desempenho Socioeconômico; e DEF = Desempenho Econômico-financeiro.

Fonte: Dados da pesquisa.

Observa-se na Tabela 11, que o Coeficiente de Correlação em ambos os casos analisados possui o valor de 0,969, evidenciando uma forte correlação entre os Rankings. Cabe destacar que a difere entre o N na correlação do Rankings do Nível de Accountability com o DS e com o DEF são os municípios que foram excluídos da amostra por falta de dados, por este fato, os valores de $\mathrm{N}$ são 60 para a correlação com DS e 54 para a correlação com DEF. Notase, também, que a siginificância a nível de $1 \%$ e coeficiente positivo na correlação analisada.

Com base nos resultados da Correlação de Kendall, infere-se que quanto maior o nível de Accountability nos municípios maior tende ser o nível de Desempenho Socioeconômico; e quanto maior o nível de Accountability nos municípios maior tende ser o nível o Desempenho Econômico-financeiro.

A partir dos achados nas correlações, o presente estudo corrobora com Campbell (1993), Gore (1995), Peters e Savoie (1996), Parker e Gould (1999), Romzek (2000), Akutsu e Pinho (2002) e Van der Hoek (2005) quanto à NPM, visto que traz inferências de quanto maior evidenciação das contas públicas, propiciando maior controle interno e externo e visando uma gestão com foco na eficiência e avaliação de desempenho, maiores serão os níveis de desempenho da gestão, resultando em melhoras nas áreas sociais, econômicas e financeiras dos entes governamentais.

\section{CONSIDERAÇÕES FINAIS}

Este estudo teve como objetivo verificar o nível de accountability nos municípios da Região Sul do Brasil, para tanto foi verificado no site das prefeituras que compõe os 10 maiores e 10 menores municípios, observando a população de cada Estado, compondo um total de 60 municípios. Para identificar o nível de transparência foi utilizada uma adaptação da metodologia de Keunecke et al. (2011) e Speck (2014) e, visando aprofundar a análise dos dados, verificou-se a correlação existente entre o
Ranking do nível de Accountability com o Ranking do Desempenho Socioeconômico e com o Ranking do Desempenho Financeiro dos municípios analisados.

Os achados demonstram que no Ranking do nível de Accountability (Tabela 5) o Estado de Santa Catarina ocupa as extremidades, ou seja, consagra-se no primeiro lugar com o município de Joinville e no último lugar com o município de Jardinópolis. A disparidade do nível de Accountability do estado de Santa Catarina faz com que, apesar de Santa Catarina possuir um município com o maior nível de evidenciação, é o Estado com menor nível de accountability da Região Sul do Brasil. Sendo o estado do Paraná o possuidor do maior nível de accountability da Região Sul do Brasil, seguido pelo estado do Rio Grande do Sul.

Destaca-se que nenhum município alcançou a pontuação máxima (47 pontos), sendo observado na coleta de dados que a maior falta de dados refere-se à não disponibilização das informações relacionadas ao patrimônio público. Observa-se, também, a falta de coesão entre dos dados apresentados entre os municípios analisados sendo que, cada município, independente do Estado que compor, terá que organizar-se para cumprir as legislações vigentes acerca da evidenciação das contas públicas em sítios eletrônicos.

Com base nos resultados da Correlação de Khendall, pode-se inferir que quanto maior o nível de Accountability nos municípios maior tende ser os níveis de Desempenho Socioeconômico e de Desempenho Econômico-financeiro. Assim, recomenda-se o cumprimento integral da legislação brasileira referente a transparência e aos princípios da NPM, visto que a maior disponibilização de informações quanto ao gestão dos recursos públicos tendem a aumentar o DS e o DEF.

Observa-se como limitação do estudo: a amostra, por não contemplar todos os municípios que compõe os Estados da Região Sul do Brasil não podendo ser generalizados os achados do estudo; 
a metodologia adotada, visto que não observa outros fatores relevantes para a informação contábil como a disponibilização de informações referente as licitações, disponibilização dos pareceres dos tribunais de contas municipais sobre as contas públicas; e a adequação às normas internacionais de contabilidade.

Para futuras pesquisas sugere-se que a amostra contemple todos os municípios da Região Sul do Brasil, bem como amplie o estudo para outras regiões do Brasil; que seja utilizada outra metodologia de coleta de dados na amostra para confirmar ou verificar outros achados, comparando-os e verificando as divergências; a análise dos fatores determinantes dos níveis de accountability nos municípios brasileiros; e as limitações desta pesquisa.

\section{REFERÊNCIAS}

Atlas do Desenvolvimento Humano no Brasil. (2015). Perfil. Recuperado de http://www.atlasbrasil.org.br/2013/pt/perfil/.

Akutsu, L., \& Pinho, J. A. (2002). Sociedade da informação, accountability e democracia delegativa: investigação em portais de governo no Brasil. Rio De Janeiro. Revista de Administração Pública. 36(5), 723-745.

Bairral, M. A. C., Coutinho e Silva, A. H., \& dos Santos Alves, F. J. (2015). Transparência no setor público: uma análise dos relatórios de gestão anuais de entidades públicas federais no ano de 2010. Revista de Administração Pública, 49(3), 642-675.

Cameron, W. (2004). Public accountability: Effectiveness, equity, ethics. Australian Journal of Public Administration, 63(4), 59-67.

Campbell, C. (1993). Public service and democratic accountability. Ethics in Public Service, 111-133.

Campos, A. M. (1990). Accountability: quando poderemos traduzi-la para o português?. Revista de administração pública, 24(2), 30-50.

Coutinho, M. J. V. (2000). Administração pública voltada para o cidadão: quadro teórico-conceitual. Revista do Serviço Público, 51(3), 40-73.

Cruz, C. F., Silva, L. M., \& Santos, R. (2009). Transparência da gestão fiscal: um estudo a partir dos portais eletrônicos dos maiores municípios do Estado do Rio de Janeiro. Contabilidade, Gestão e Governança, 12(3), 102-115.

Gore, A. (1995). Common Sense Government. Works Better and Costs Less. Third Report of the National Performance Review. US Government Printing Office, Superintendent of Documents, Mail Stop: SSOP, Washington, DC.

Hair, J., Babin, B., Money, A., \& Samouel, P. (2005). Fundamentos de métodos de pesquisa em administração. Porto Alegre: Bookman Companhia Ed.

Hood, C. (1995). The "New Public Management" in the 1980s: variations on a theme. Accounting, organizations and society, 20(2-3), 93-109.

Instituto Brasileiro de Geografia e Estatística (IBGE). (2015). Cidades. Recuperado de http://www.cidades.ibge.gov.br/xtras/home.php.

Keunecke, L. P., Teles, J., \& Flach, L. (2011). Práticas de Accountability: uma análise do índice de transpa-rência nos municípios mais populosos de Santa Catarina. Revista Contemporânea de Contabilidade, 8(16), 153-174.

Klein, A. Z., Silva, L. V., Machado, L., \& Azevedo, D. (2015). Metodologia de pesquisa em administração: uma abordagem prática. São Paulo: Atlas.

Lapsley, I. (1999). Accounting and the new public management: instruments of substantive efficiency or a rationalising modernity?. Financial Accountability \& Management, 15(3-4), 201-207.

Lei Complementar no 131, de 27 de maio de 2009. (2009). Estabelece normas de finanças públicas voltadas para a responsabilidade na gestão fiscal e dá outras providências, a fim de determinar a disponibilização, em tempo real, de informações pormenorizadas sobre a execução orçamentária e financeira da União, dos Estados, do Distrito Federal e dos Municípios. Recuperado de http://www.planalto.gov.br/ccivil_03/leis/LCP/Lcp131.htm.

Leite Filho, G. A., Colares, A. F. V., \& Andrade, I. C. F. (2015). Transparência da gestão fiscal pública: um estudo a partir dos portais eletrônicos dos maiores municípios do Estado de Minas Gerais. Contabilidade Vista \& Revista, 26(2), 114-136

Nakagawa, M. M., Relvas, T.R.S., Dias Filho, J. M. (2007). Accountability: a Razão de ser da Contabilidade. Revista de Educação e Pesquisa em Contabilidade. 1(3), 83-100.

O'Donnell, G. (1998). Accountability horizontal e novas poliarquias. Lua nova, 44(98), 27-54

Pérez-López, G., Prior, D., \& Zafra-Gómez, J. L. (2015). Rethinking new public management delivery forms and efficiency: long-term effects in Spanish local government. Journal of Public Administration Research and Theory, 25(4), 1157-1183.

Parker, L. D., \& Gould, G. (1999). Changing public sector accountability: critiquing new directions. Accounting forum. 109-135.

Peters, B. G., \& Savoie, D. J. (1996). Managing incoherence: The coordination and empowerment conundrum. Public Administration Review, 281-290.

Perci, A., Pieranti, O. P., \& Rodrigues, S. (2008). Governança e New Public Management: convergências e contradições no contexto brasileiro. Organizações \& Sociedade, 15(46), 39-55.

Pereira, L. C. B. (1996). Da administração pública burocrática à gerencial. Revista do Serviço público, 47(1), 07-40.

Pinho, J. A. G. D., \& Sacramento, A. R. S. (2009). Accountability: já podemos traduzi-la para o português?. Revista de Administração Pública-RAP, 43(6), 1332-1368.

Platt Neto, O. A., Cruz, F. D., Rolim Ensslin, S., \& Ensslin, L. (2007). Publicidade e transparência das contas públicas: obrigatoriedade $e$ abrangência desses princípios na administração pública brasileira. Contabilidade Vista \& Revista, 18(1), 75-94.

Pollitt, C., Van Thiel, S., \& Homburg, V. (2007). New public management in Europe. Basingstoke: Palgrave Macmillan.

Programa das Nações Unidas para o Desenvolvimento Humano (PNUD). (2015). Desenvolvimento Humano e IDH. Recuperado de http://www.pnud.org.br/IDH/IDH.aspx?indiceAccordion=0\&li=li_IDH.

Ramos, M. P., \& Schabbach, L. M. (2012). O estado da arte da avaliação de políticas públicas: conceituação e exemplos de avaliação no Brasil. Revista de administração pública. 46(5), p. 1272-1294.

Raupp, F. M., \& de Pinho, J. A. G. (2013). Accountability em câmaras municipais: uma investigação em portais eletrônicos. Revista de administração, 48(4), 770-782.

Rocha, A. C. (2013). A realização da accountability em pareceres prévios do Tribunal de Contas de Santa Catarina. Revista de Administração Pública, 47(4), 901-926.

Romzek, B. S. (2000). Dynamics of public sector accountability in an era of reform. International review of administrative sciences, 66(1), 21-44.

Sacramento, A. R. S., \& Pinho, J. A. G. (2007). Transparência na administração pública: o que mudou depois da lei de responsabilidade fiscal? um estudo exploratório em seis municípios da região metropolitana de salvador. Revista de Contabilidade da UFBA, 1(1), 48-61.

Secchi, L. (2009). Modelos organizacionais e reformas da administração pública. Revista de Administração Pública. 43(2), 347-369.

Secretaria do Tesouro Nacional. (2012). Manual de contabilidade aplicada ao setor público: aplicado à União e aos Estados, Distrito Federal e Municípios / Ministério da Fazenda, Secretaria do Tesouro Nacional (5 ed.). Brasília: Secretaria do Tesouro Nacional, Coordenação-Geral de Contabilidade.

Schillemans, T. (2015). Calibrating Public Sector Accountability: Translating experimental findings to public sector accountability. Public Management Review, 18(9), 1400-1420

Schwella, E. (2005). Inovação no governo e no setor público: desafios e implicações para a liderança. Revista do Serviço Público, 56(3), 259-276.

Speck, B. (2014) Metodologia do Índice de Transparência. Recuperado de http://www. http://indicedetransparencia.com/metodologia-2014.

Van Der Hoek, M. P. (2005). From cash to accrual budgeting and accounting in the public sector: The Dutch experience. Public Budgeting \& Finance, 25(1), 32-45. 